\title{
Specularity, brightness, achromatic color-and orthogonality
}

\author{
HOWARD R. FLOCK and STEVEN NUSINOWITZ \\ York University, North York, Ontario, Canada
}

\begin{abstract}
For objects under the same illumination, the more specular object appeared brighter. This occurred irrespective of an object's apparent achromatic color, the distance at which it was viewed, the level of illumination, the method used for collecting observations, and other conditions. Also, when identical specular objects were differently illuminated, the one under the higher illumination, with the higher maximal luminances, appeared brighter. In the five experiments, which involved 340 subjects subdivided into 17 groups, large fields of view and real spaces were used. The results supported the conclusion that apparent brightness and achromatic color are orthogonal phenomena, and that in four of these experiments increased apparent brightness was correlated to, if not determined by, maximal specular luminances without regard to achromatic colors and diffuse luminances. This conclusion was necessarily modified by a fifth experiment, which showed that if placed under sufficiently high levels of illumination, less specular surfaces appeared brighter than more specular surfaces. This was taken to mean that a total account of the apparent brightness of surfaces would depend on an undiscovered algorithm involving maximal specular and diffuse luminances and their areal extents. With regard to the validity of the studies, the subjects were shown to have phenomenally discriminated brightness from glossiness and glare. Finally, phenomenal gloss and glare were found to be correlated to a surface's level of specularity.
\end{abstract}

Objects with specular highlights appear brighter than objects without such highlights, or so it has seemed to us in informal observations. In our office, for example, the gray metallic file cabinet, the black telephone casing, the bottled india ink, the shiny gray leather on a chair, and other specular objects appear brighter than other surfaces, brighter even than various white surfaces that are not specular. We placed two pieces of black coal in the room, one polished (specular), one unpolished (matte). Both appeared black. But the polished piece appeared brighter than its unpolished counterpart, and, in fact, brighter than most other surfaces in the room. We selected two grays that appeared to be the same color. The more specular of the two grays appeared brighter. In other words, with everything under the same overall illumination, the more specular surfaces appeared to be brighter than the less specular and matte surfaces.

Is this informal phenomenology with regard to specular surfaces generally true? Does an achromatically colored surface continue to appear to have approximately the same color, but to be apparently brighter when its specularity is increased? Helmholtz (1925) might possi-

This research was supported in part by grants from the Natural Sciences and Engineering Research Council of Canada and by two Minor Research grants from the Faculty of Arts, York University. We wish to thank the following students for their assistance in running the experiments: Andrea Birkenfeld, Sheila Gallagher, Nicholas Goboly, Roswitha Roese, Eric Rubel, and Frank Scrivo. We also wish to thank Donald Mershon for building the apparatus described in Experiment 1. Finally, we wish to thank Stuart Anstis, Ian Howard, Tony Nield, and Martin Regan for their helpful reading of the manuscript. Requests for reprints should be sent to Howard R. Flock, Department of Psychology, York University, Downsview, ON M3J IP3, Canada. bly have said no. The brightness of surfaces, from not very bright to dazzling bright, and the achromatic colors, from the blackest black imaginable through the grays to the whitest white imaginable, are, Helmholtz claimed, unidimensional phenomena, perfectly and positively correlated. A surface that appears brighter than a second surface should also appear lighter in color. And a surface that appears lighter in color should also appear brighter. (See Heggelund, 1974, for a review of Helmholtz's unidimensionality.) In comparison, our informal observations imply that apparent brightness and achromatic color are not only separate phenomenal dimensions (bidimensional), but are also independent and orthogonal. For the experiments described in this paper, therefore, we had two major goals: (1) to demonstrate that more specular surfaces appear brighter than less specular surfaces, when both are under the same illumination, and (2) to demonstrate that apparent brightness and achromatic color are independent orthogonal phenomena (at least for the levels of photopic illumination used in our experiments).

\section{The Stimulus for Apparent Achromatic Colors}

If apparent achromatic color and brightness are independent, then the two phenomena will be specified by different stimuli. The appearance of achromatic color is determined, according to numerous theorists, by luminance ratios (also called light gradients and ratios of light intensities) among areal regions of light. Some early theorists who held this view were Helson $(1938, \mathrm{pp} .450$, 461-463; 1943, pp. 561-564), Kardos (1934, pp. 84-86), Koffka (1932, especially pp. 184-185; 1935, pp. 244- 
249), and Wallach $(1948,1963)$. Luminance ratios were sometimes referred to as ratios of albedos (e.g., by Henneman [1935, pp. 52-53, 74], who also on pp. 77-80 accepted Koffka's views with regard to luminance ratios and their achromatic effects). In general, for flat, matte surfaces under the same illumination, albedo ratios will correctly specify luminance ratios. Luminance ratios were sometimes also referred to as contrast ratios (e.g., by Beck, 1964; Flock, 1974; Flock \& Noguchi, 1970).

Helson's $(1938,1943,1964)$ formulation of luminance ratios was unique in an important respect. He formed the ratio from a region's luminance and from a weighted average of luminances for the entire visual field. In making that step, Helson moved the discussion away from the artificiality of displays and scenes with only a few different luminance regions to the naturalness of real scenes, with their complexly configurated luminances.

The effect of luminance ratios on apparent achromatic colors was demonstrated in a recent set of experiments (Flock \& Nusinowitz, 1984). Observers were allowed a wide-angle binocular view of a small furnished space. By varying the position of a light source, black matte walls in the small room were made to appear any color from white to deep black. The changes in illumination differentially altered the luminances of the walls. That changed the luminance ratios among the various walls. With those changes in luminance ratios, the apparent achromatic colors of the black walls correspondingly altered.

In explaining achromatic appearances, Koffka (1932) adopted a concept of appurtenance-that, for example, spatial coplanarity of surfaces was particularly pertinent to the achromatic appearances of those surfaces. Flock and Nusinowitz (1984, pp. 127-129) also adopted a concept of appurtenance, but they rejected spatial arrangements, such as the coplanarity of surfaces, as a main criterion for that appurtenance. In their experiments, Flock and Nusinowitz (1984) found that spatial arrangements had little to do with achromatic appearances, unless the spatial arrangement altered the surface's luminance. As a result, they adopted the view, apparently unique to them, that luminance ratios were formed among surfaces that "are seen to be illuminated by the same sources or to derive or generate their light in the same way. Provided the surfaces have some spatial coherence, it doesn't matter how they are arranged spatially" (Flock \& Nusinowitz, 1984 , p. 127). (The concept "seen to be illuminated by the same sources" enables one to explain phenomena like those of Gelb [1929] and Gilchrist [1980, Figure 5]. In Gilchrist's experiment, for example, achromatic appearances changed, we believe, because of the change in the seen illumination of the target, not because of the coplanarity produced by its change in spatial location, as Gilchrist argued. The change in spatial location functioned to move the target into a differently illuminated space. The luminance ratios in that illuminated space then determined the achromatic colors of the surfaces, irrespective of the spatial relations.)

Nothing cognitive was implied by this concept of the "seen" illumination. In fact, local adaptation mechanisms in the eye, like those described by, for example, Werblin (1973), might perhaps function to specify which surfaces were being illuminated commonly and were therefore "appurtenant."

In the experiments in this paper, we used small threedimensional rooms with objects in them. Subjects, viewing monocularly or binocularly, were given a wide-angle view of the rooms. Each scene was illuminated naturally by one or two light bulbs or by an extended source. For these conditions, all of the surfaces in a scene were, we assumed, "appurtenant" (seen to be illuminated by the same sources of light). Hence, ratios of their nonspecular luminances would account for the prevailing achromatic colors.

\section{The Stimulus for Apparent Brightness}

An appearance of brightness unarguably refers to the intensive phenomena associated with the energy levels of primary sources of light. The International Commission on Illumination (CIE), for example, has adopted as standard vocabulary the definition that brightness is an "attribute of visual sensation according to which an area appears to emit more or less light"' $(1970$, p. 118). The specular reflections or highlights that are to be found on most surfaces constitute such a stimulus for the apparent brightness of the surface. Specular reflections are, after all, mirror images of the light sources in the scene, although admittedly such images are highly degraded in natural scenes (Flock, 1984). In registering specular reflections, whether they be degraded mirror images or not, one is, in effect, looking at the light sources and experiencing their intensive effects. The apparent brightness of the overall surface would then be correlated, according to our experimental hypotheses, with the intensity level of its highlights. Since there are no demonstrations in the empirical literature of such correlations (although they were expected by Beck [1972, p. 13] and were implied by Kozaki [1973]), we shall attempt, in this paper, to demonstrate them.

To be quite explicit on this matter of apparent brightness, look at the highlights on some black specular object, such as a telephone. As we observed earlier in our informal phenomenology, the highlights and the surrounding surface that is the setting for the highlights appear bright. Then, as the luminances of the highlights are reduced toward zero, the brightness of both the highlights and the surrounding surface appears to decrease.

Specular and diffuse luminances. To clarify what occurs when specular highlights are increased (or decreased) in intensity, consider the interaction of light with matte and with nonmetallic specular surfaces. These different interactions define a distinction between diffuse and specular reflectances as properties of a surface and diffuse and specular reflections as light stimuli.

A nonspecular (matte) surface scatters the rays of light that impinge, thereby producing what is called a diffuse reflection. A specular surface reflects light rays directionally, rather than scattering them, which accounts for the much greater intensity of the specular reflection. When 
a surface is made smoother (by being polished or treated in some way) or is replaced by one that is naturally smoother, specularity is correspondingly increased. For a given level of specularity, the intensity of the specular reflection will depend not only on the surface's specularity, but also on such factors as the intensity of the illuminating source, the surface's refractive index, and the angle of incidence of the illuminating light rays.

Specular reflections (viz., mirror images of primary and secondary illuminating sources) are detectable at and near the specular angle, which is the angle oppositely signed to the angle of incidence of light rays on the surface. At other angles of view, one detects the diffuse, nonspecular reflection from the surface, which is like the diffuse reflection (luminance) of a "matte" surface. Also like matte surfaces, the diffuse reflection of the specular surface carries information about the surface's achromatic color (and its chromatic color and texture).

Specular highlights (reflections) on a surface can be made less detectable by varying one or more conditionsfor example, by moving the viewing point away from the specular angle; changing the angle of incidence of the light; changing the plane of the surface by making it flatter or reorienting it; reducing surface specularity (the method used mostly in this paper); substituting a surface with a smaller refractive index; and increasing surface albedo from a low value (low reflectance, high absorption, as with blacks) to a high value (high reflectance, low absorption, as with whites). Increasing albedo homogenizes surface luminances. This homogenizing occurs because of the increased secondary reflections on and between surfaces, the decreased particle sizes below the first surface, and possibly the decreased difference between a surface's specular and diffuse reflections. Finally, reducing illumination also minimizes the detectability of specular highlights.

Despite their presence on most surfaces, specular highlights are rarely allowed to intrude into visual displays and theory. As just one example of this avoidance, in setting forth retinex theory, Land and McCann (1971) described, in their opening paragraphs, the precautions they took to decrease the probability of detecting specular highlights. Flat, matte, untextured surfaces were used. Very low albedos were avoided. The light source was at $45^{\circ}$ and the surface was viewed at an angle normal to the surface.

As a result of such avoidances of specular highlights, the empirical literature concerned with the apparent brightness of surfaces has been predominantly about matte surfaces.

The apparent brightness of matte surfaces. When specular images have been excessively degraded and are virtually undetectable on a surface, the surface is said to be "matte." According to our view, when specular luminances reach such "zero levels," apparent brightness, as derived from them, must also reach zero. For these conditions, light sources are no longer imaged wherever the gaze falls. Despite the virtual disappearance of specu- lar reflections, the matte surface does not lack in an appearance of brightness, although its surface may have become phenomenally dull. In a uniformly illuminated scene, "matte" surfaces, we claim, appear less bright than more specular surfaces, but they do appear bright. They do not appear "zero-bright"' (unless "dull" is construed to be one form of "zero-bright"). Also, if two surfaces are equally matte (or equally specular), but are differently illuminated, the one under higher illumination, with its higher luminances (or with its more intense highlights) might be expected to appear brighter.

This description of the brightness of matte surfaces means that specular reflections do not give a complete account of the apparent brightness of surfaces. We propose, however, that matte surfaces follow virtually the same rule as for specular surfaces. Consider first the "rule" for specular surfaces.

The specular highlight is an intensive deviation from the luminance level of its adjacent surround. The amount of deviation specifies, we have argued, the apparent brightness of both the highlights and the adjacent surface in which the highlights are embedded. As the specular luminance is reduced, its phenomenal brightness and that of its surrounding surface decrease.

The same rule may be applied to matte surfaces in a uniformly illuminated scene. Although spatially discontinuous, the matte surfaces, we shall assume, appear similar in brightness, irrespective of their achromatic colors. That is, the matte surface with the highest luminance (which in a real scene is usually a white surface) would appear at some level of brightness, depending on its intensity and on adaptive mechanisms in the eye. Adjacent matte surfaces, irrespective of their achromatic color, would then, from one to the other, appear similar in brightness, although not necessarily as bright as the region of maximal luminance. This hypothesis has support from, for example, Koffka (1932 and 1935, p. 247). In explaining Gelb's phenomenon, Koffka asserted that the black disk and the white slip of paper held against it, both seen in the same strong illumination, appeared equally bright. Kozaki (1973, Figure 4B), also, showed that achromatic colors from black to light gray appeared equally bright when presented against a white background. Approximately the same view may have been espoused by Evans (1964, p. 1469). By refusing to attribute brightness to achromatically colored surfaces, Evans may have been saying that those surfaces, from black to light gray, appeared equally bright.

When matte surfaces are uniformly illuminated, the brightness function for surfaces from black to white might have some rise. Nevertheless, if Evans (1964), Koffka (1935), and Kozaki (1973) were even approximately correct in their theoretical expectations, the rise would be expected to be slight.

In this explanation of the brightness of matte surfaces, there is no separate image of the light source, as given by specular highlights. Because of that, the explanation of the brightness of matte surfaces is not as parsimonious 
as the explanation for the brightness of specular surfaces. Nevertheless, in the absence of specular highlights, perhaps the visual system treats the matte region of highest luminance (or even some average luminance for part of a scene) as a "surrogate" image of the source, or at least as an indicator of the overall illumination.

\section{THE EXPERIMENTS}

We have argued the independence of the visual phenomena of achromatic color and brightness. To demonstrate that independence and other expectations developed above, we ran five experiments. In the first, both the orthogonality of achromatic color and its brightness and the claim that a more specular surface will appear brighter than a less specular surface were examined. Pairs of matte and specular surfaces, from black to white, were the stimuli. Each pair had first been matched for achromatic color. The surfaces were individually presented in a three-dimensional space and were viewed monocularly with a large field of view. The two achromatically matched surfaces in a pair, each under the same high illumination, were judged as to their brightness (one group of subjects) and their achromatic color (a second group of subjects). The experiment was then replicated for low illumination, using two more groups of subjects. In Experiment 2, conditions differed, but the objectives of the experiment were the same as in Experiment 1.

For Experiment 3, from the two levels used in Experiment 1 , specularity was increased to four different levels. The four specular surfaces, matched in blackness, were presented simultaneously in pairs and judged as to which one of the pair appeared brighter. The experiment was replicated for light gray surfaces, with the intention of determining the generality of the correlation between apparent brightness and specularity.

For Experiments 1 to 3, the surfaces that were compared were always under the same overall illumination. For those conditions, a more specular surface might appear brighter because it was more specular or because it had higher maximal luminances. The purpose of Experiment 4 was to decide between those two possibilities. Surfaces were matched for achromatic color and specularity and were placed under different levels of illumination. If level of specularity alone determines apparent brightness, then both surfaces should appear equally bright. On the other hand, if higher maximal luminances determine apparent brightness, then the more highly illuminated surface should appear brighter.

If, in Experiment 4, the matched surface under higher illumination turned out to appear brighter, that could occur because it had not only more intense highlights but also higher diffuse luminances. The latter possibility would imply a general role for diffuse luminances in the determination of the apparent brightness of surfaces.

One aspect of this possibility was investigated in Experiment 5 . Less specular surfaces were placed under higher illumination; more specular surfaces were placed under lower illumination. The two scenes were otherwise identical. For those conditions, the more specular surfaces possessed the highest maximal luminances, but the less specular surfaces possessed the highest diffuse luminances and probably had the highest overall average luminances. If the surfaces with the highest diffuse luminances but lower maximal luminances appeared brighter, then maximal luminances would not necessarily be the deciding factor with regard to which surface appeared brightest.

Resolution of the issues explored by Experiment 5 would start to clarify the relationship between apparent brightness, on the one hand, and illumination, specularity, maximal luminances, diffuse luminances, and overall average luminances, on the other. (In this paper, the concept "overall average luminance" has been generally avoided. In our displays, luminances often varied by large amounts over small areas. We do not know how visual systems weight such different luminance intensities in generating their output. Under most of our conditions, therefore, we were hesitant to make claims about overall average luminances.)

Finally, throughout Experiments 3 to 5, data were collected with respect to the use of the term apparent brightness. For the conditions being used, we wished to establish whether observers could reliably distinguish among a number of phenomenal characteristics of surface. Specifically, we wished to discover if they would be discriminative in using the terms brightness, glossiness, and glare.

\section{Experiment 1}

The purpose of Experiment 1 was to show that specular objects appear brighter than achromatically matched matte objects, even when the matte and specular objects appear to be the same achromatic color. This was tested for five different levels of achromatic color, from black to white, for objects at two different distances, and for two levels of illumination. Viewing was monocular, with a wide-angle view of a real space.

\section{Method}

Subjects. The 84 subjects were volunteers from introductory courses in psychology at York University. All subjects had 20/20 vision in the right eye, either uncorrected or corrected with contact lenses, and all were naive with regard to the experiments. Median age was 19 years.

Apparatus. A round $51^{\circ}$ monocular aperture was mounted on the front wall of an enclosed space that was $91 \mathrm{~cm}$ high, $67 \mathrm{~cm}$ wide, and $125 \mathrm{~cm}$ deep. An aperture for the right eye was positioned $2 \mathrm{~cm}$ in from the left wall and $36 \mathrm{~cm}$ above the floor of the little room. The aperture was oriented to the right and downward, and excluded any view of the left wall and the ceiling. Visible were the lower $60 \%$ of the back and right walls and $75 \%$ of the floor (or approximately $18 \mathrm{cu} \mathrm{ft}$ of space). Separate opaque and translucent shutters were built into the eyepiece.

The right wall and floor of the little room were covered with a matte wall paper, composed of intricate black, gray, and white figures. The equivalent Munsell neutral $(N)$ values of these figures were $3.25,7.75$, and 9.25 , respectively. (For this experiment and all others in this paper, the achromatic colors were approximately 
neutral and were matched to neutral Munsell samples.) The left and rear walls of the room were covered with white fabrics (Munsell N9.3).

The room was illuminated from above by a bank of seven GE F-20 T-12-D daylight fluorescent tubes, each $57 \mathrm{~cm}$ long, spaced $7.5 \mathrm{~cm}$ apart from center to center. The total extended light source was $57 \mathrm{~cm}$ wide and $48 \mathrm{~cm}$ deep, located with its back edge $22 \mathrm{~cm}$ from the far wall and its forward edge $55 \mathrm{~cm}$ from the front viewingwall. Also, it virtually spanned the width of the room. Below the light source, $91 \mathrm{~cm}$ from the floor of the room, was a thin sheet of translucent diffusing material that was supported by a thin sheet of glass. The illumination produced by these arrangements was called the high-illumination condition. When a large neutral-density filter, $67 \mathrm{~cm}$ wide and $80 \mathrm{~cm}$ long, was placed just below the light source, illumination was reduced by a factor of $1 / 40.2$. That produced the low-illumination condition. Luminance measures given in the description of this experiment, unless otherwise indicated, are for the high-illumination condition. Dividing any of those measures by 40.2 will give luminance values for low illumination. All measures were made in foot-lamberts, using a Spectra Pritchard photometer with a 6-min probe.

Under high illumination, some luminance measures for various visible surfaces in the room were as follows. The white figure in the pattern on the floor had a luminance value of approximately $120 \mathrm{fL}$, virtually everywhere on the floor. (A Munsell N9.5 sample, on the floor, had a luminance of $126.5 \mathrm{fL}$.) Dividing $120 \mathrm{fL}$ by 1.70 or 10 gives the luminances of the gray and black figures on the floor, or 70.5 and 12 , respectively. The rear wall, a curtain with folds, gave a range of values from $20 \mathrm{fL}$ within folds to a maximum of $70 \mathrm{fL}$. The white figures on the right wall had luminances from $68 \mathrm{fL}$ low on the wall to $100 \mathrm{fL}$ high on the wall. The lu- minance of the adaptation field in the shutter of the viewing aperture was $50 \mathrm{fL}$.

Test fields. Each of 10 cylinders, $9 \mathrm{~cm}$ high and $5.3 \mathrm{~cm}$ in diameter, and cut from solid maple, was wrapped in a different Munsell neutral paper, five with matte paper and five with specular (glossy) paper. The tops of the cylinders were left uncovered and were visible to the subjects. Except for the two blacks, the Munsell values were the same for the paired matte and specular test fields (TFs). The paired neutral Munsell values were: 9.5 (white), 7.0 (light gray), 5.5 (middle gray), 4.0 (dark gray), 1.5 (matte black), and 0.5 (specular black).

Each of the $10 \mathrm{TFs}$ was separately presented to the observer at two locations in the room, a total of 20 randomly ordered trials. A cylinder in the far position, $125 \mathrm{~cm}$ from the observer, was slightly forward of the far right corner, $8 \mathrm{~cm}$ from the right wall, $20 \mathrm{~cm}$ in front of the rear wall, and angularly measured $2.3^{\circ} \times$ $4.2^{\circ}$, for the base and height, respectively. A cylinder in the near position, $70 \mathrm{~cm}$ from the observer, was at the front of the visible part of the room, slightly to the left side of an observer's field of view. It was $41 \mathrm{~cm}$ from the right wall, $69 \mathrm{~cm}$ in front of the rear wall, and angularly measured $4.4^{\circ} \times 7^{\circ}$ for the base and height. In the near position, the surface of a cylinder was just below the forward edge of the extended light source. In that position, its visible front surface received less light than the same TF in the far position. The surround luminances, however, were about the same, regardless of the position of the TF. Because of these different luminance relations between a TF and its surrounds, achromatic color might be expected to (and did) appear darker in near TFs than in far TFs. (See Results section.)

Light measures for the five matte and five specular TFs, under high illumination at the near and far positions, are summarized in

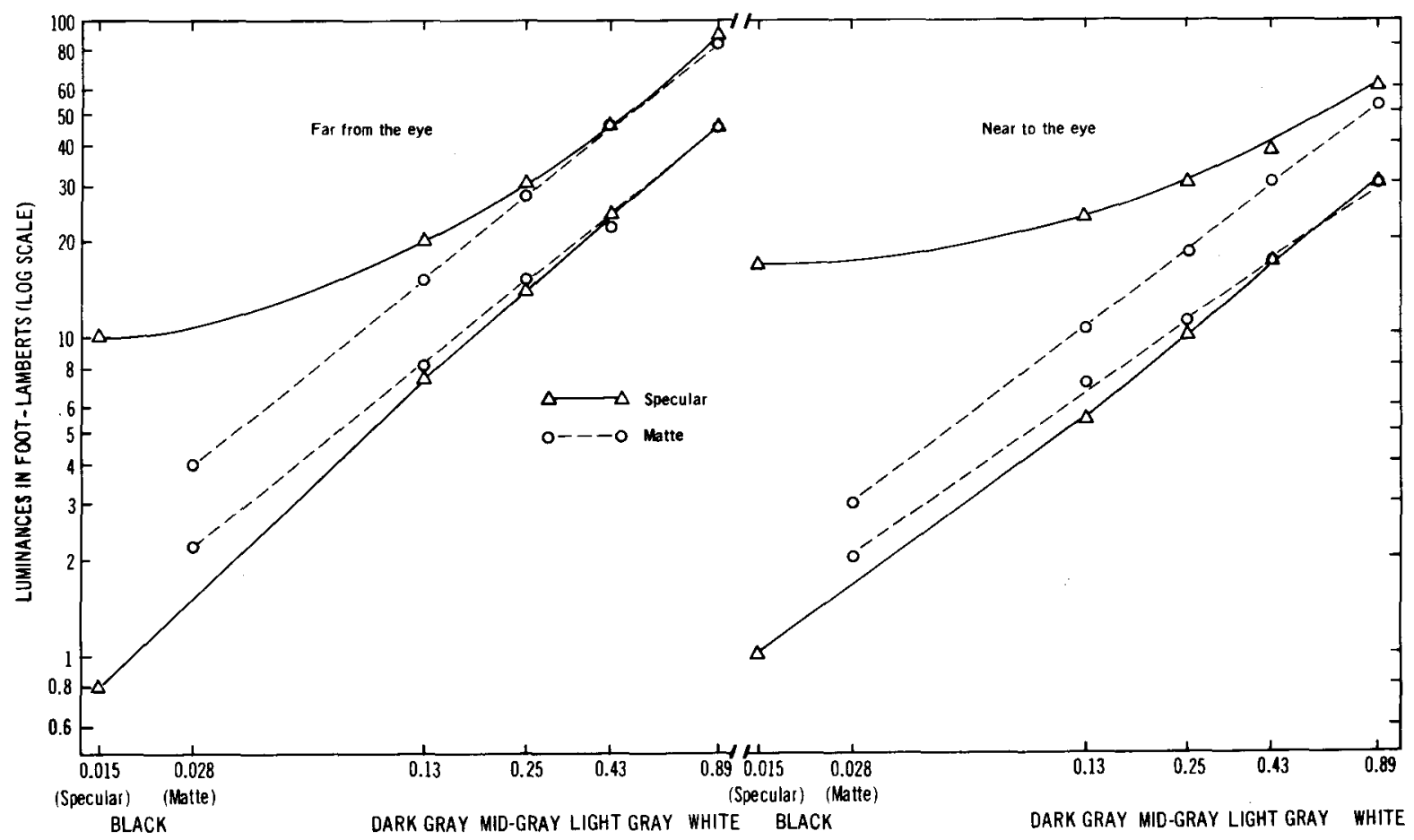

REFLECTANCES OF NEUTRAL MUNSELL SURFACES ON A LOG SCALE

Figure 1. Maximum and minimum luminance values for 10 achromatically neutral surfaces, five specular $(\Delta-\Delta)$ and five matte $(\mathrm{O}---\mathrm{O})$, under high illumination. Left half, far from the eye; right half, near to the eye. In each half of the graph, the upper of two solid and two dashed lines are maxima; the lower of a pair are minima. 
the log-log plot in Figure 1. The corresponding functions for low illumination were virtually identical, except that the $y$-axis was recalibrated for the lower illumination. The reflectances of the five TFs, varying form 0.015 to 0.89 , are given on the abscissa; luminances, varying from 0.80 to $100 \mathrm{fL}$, a range of 125 to 1 , are given on the ordinate. The ordinate for low illumination varied from about 0.022 to 2.2 , a range of 100 to 1 .

The luminances in Figure 1 were recorded from the upper twothirds of a TF's surface area. In each half of Figure 1, there is a pair of dashed straight lines for matte surfaces and a pair of solid lines for specular surfaces. The top and bottom lines in each pair describe maximal and minimal light values, respectively, for each of the TFs.

In inspecting the functions in Figure 1, one should keep in mind some limitations associated with the probe of the photometer. First, the probe was relatively large in angular size ( $6^{\prime}$ of arc), given the tiny angular size of the specular "hot spot" that was being measured. In a particular measure of a specular image, the probe picked up light from more than, for example, just an imaged white figure. It was also averaging the light from imaged black and gray figures and from the achromatic color of the TF itself. When the TF objects were near, this problem was only slightly diminished. Second, the specular TFs were only "moderately" specular. Hence, because of the size of the probe, the luminance of a specular highlight would be some kind of weighted sum of both the diffuse and specular reflections. The ratio of luminances on a TF surface, as between its maximum and minimum, is given in Figure 1 by the vertical separations between a pair of dashed or solid lines.

Apart from the research in this paper, Figure 1 might be of interest in and of itself. It is frequently difficult to gain access to systematic measures of specular surfaces like those in Figure 1. However, Hunter (1975) gathered together from various sources and published data on a limited number of luminance functions for matte and specular surfaces. Although his functions had more precision than those in Figure 1, they were also more limited in scope.

Procedure. The effects of five variables were investigated. There were five levels of achromatic color, black, dark gray, middle gray, light gray, and white; two levels of specularity, matte and specular; and two distances, near and far. These three variables were "within" variables. They produced 20 experimental trials (5 colors $\times 2$ surfaces $\times 2$ distances), which were randomly reordered and presented to each subject. In addition, there were two "between" variables: two different instructions, to judge achromatic color (one group) and brightness (a second group), and two levels of illumination, high and low. This gave a total of four "between" groups of subjects-two levels of instructions times two levels of illumination.

In the training session, the subject viewed the $10 \mathrm{TFs}$ presented on a tray in the small room. These TFs were ordered in two rows, from white on the left to black on the right. The five specular TFs in the front row only slightly obscured parts of the five matte TFs in the back row. While the subject was inspecting the TFs, he/she was asked: (1) Do you see surfaces that appear white? Appear gray? Appear black? (2) Do you see surfaces that appear brighter than other surfaces? Less bright than other surfaces?

Brightness groups. Each subject in the two brightness groups was next asked to select from among the 10 TFs on the tray the TF that "appeared to be the brightest of all, the least bright of all, and middle bright." (Some of the results of these comparisons are given below in the Results section.) The numbers 19,11 , and 15, respectively, were assigned to those TFs. The subject was told to memorize the exact brightness appearance of those surfaces and the numbers that had been assigned to them, ignoring the achromatic color of the TF. They were told that they were to be individually shown 20 different TFs that would vary in brightness from less bright to brighter than any they had seen so far. (In fact, the 10 matte and specular TFs were presented individually to them, near and far, under the illumination used during training.) The subjects were told to use the numbers 10 to 20 , including one-place decimals, a scale of 101 gradations, to indicate the precise brightness of each surface that they were to see. This training session lasted about 4 min.

During the experiment, the subject was given $10 \mathrm{sec}$ for each of the 20 judgmental trials. Between each of the 20 trials there was a 15-sec period of light adaptation, at the light level of the scene.

Achromatic-color groups. The continuing instructions for the two achromatic color groups were as much as possible like those for the brightness groups. Each subject was asked to select from among the 10 TFs on the tray the TFs that appeared the whitest, the lightest gray, the darkest gray, and the blackest. The numbers 21,19 , 11 , and 9, respectively, were assigned to those TFs. Other procedures and additional training were exactly like those for the brightness group, except for the following differences. The subjects were told to ignore the brightness of the TF and to attend only to its achromatic color. They were told to use the numbers 8 to 10 for blacks, 10 to 20 for grays, and 20 to 22 for whites, including one-place decimals, a scale of 141 gradations, to indicate the precise achromatic color of each of the 20 surfaces.

For high illumination, there were 28 subjects in each of the two instruction groups. For low illumination, which was run later, there were 18 subjects in the brightness group and only 10 in the achromatic-color group. These reductions in group size were deliberate and based on the reliability of the data under high illumination. Finally, each of the four groups of subjects was divided equally between men and women.

\section{Results}

Brightness. The brightness results are shown in Figure 2. To assess those results, consider first Figure 2A for high illumination. It shows mean brightness judgments of the five TFs, for specular, matte, near, and far conditions. One feature in the figure is particularly prominent and relevant. All data points for specular surfaces (upper two lines in Figure 2A) fall above every data point for the matte surfaces (lower two lines). Moreover, the least bright specular surface appeared significantly brighter than the brightest matte surface. That fact is extremely persuasive in indicating that specular surfaces appeared brighter than matte surfaces. The main effect of the separation between the functions for matte and specular surfaces was significant $[F(1,27)=87, N=28, p<.001]$. Also, the specular surface that appeared least bright, the light-gray TF in the far position with a mean brightness judgment of 14.9, was judged brighter than the brightest appearing matte surface, the white TF in the far position with a mean brightness judgment of 13.5. The frequency of 20 to 7 with one tie had a binomial chance probability $(p<.02)$.

Approximately the same results, although with somewhat more noise, occurred in Figure $2 B$ for brightness judgments under low illumination. The main effect for matte and specular surfaces was significant $[F(1,27)=$ $32, N=18, p<.001]$. In addition, the specular surface that appeared least bright was chosen more frequently as appearing brighter than was the brightest appearing matte surface.

The finding that specular objects appeared brighter than matte objects far exceeded our initial expectations. The initial hypothesis was that the more specular of an achro- 
A : HIGH ILLLIMINATION

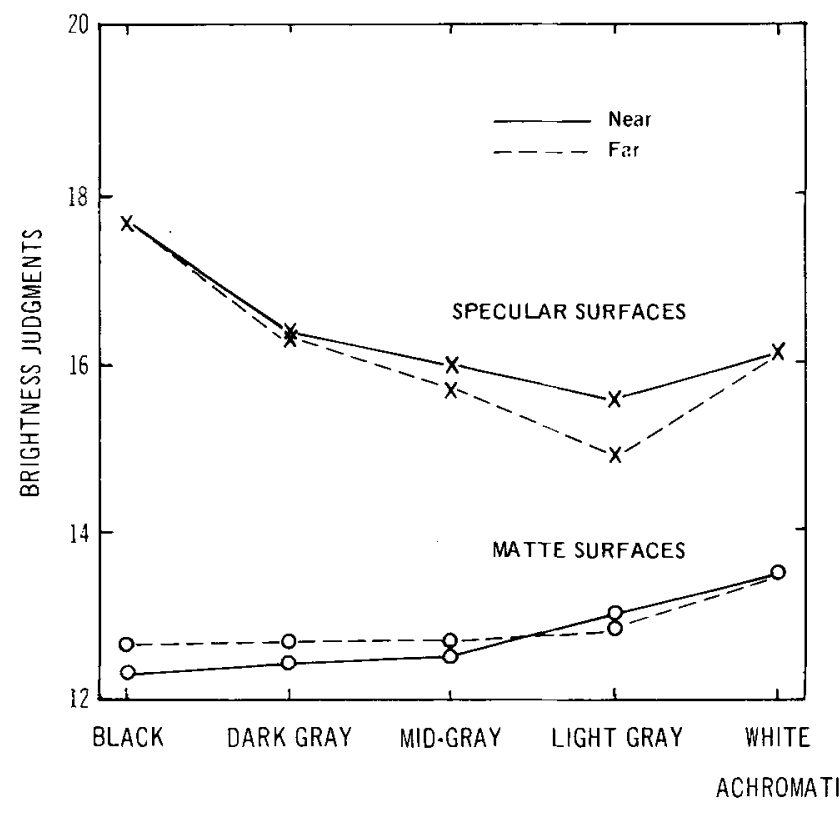

B: LOW ILLUMINATION

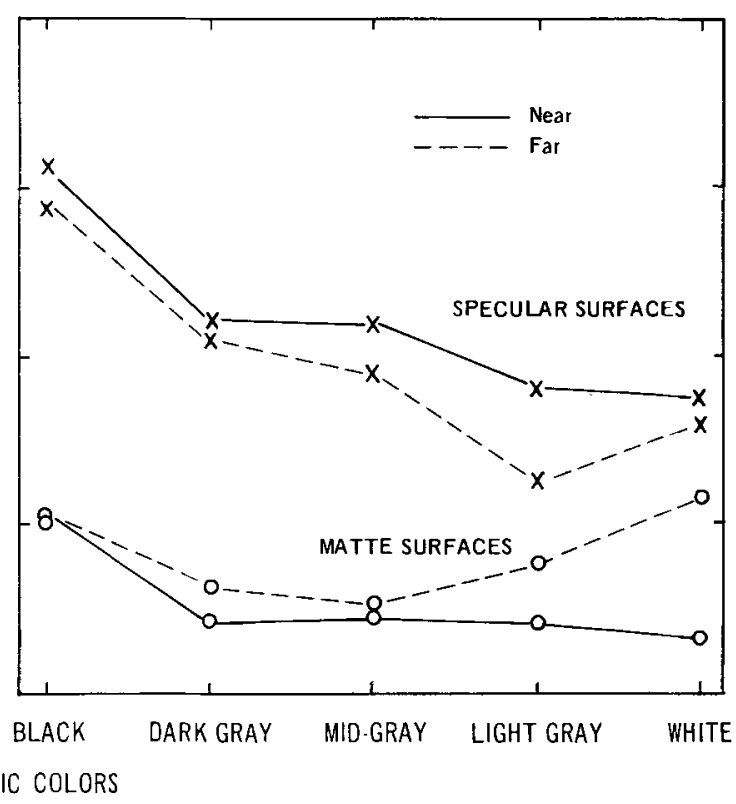

Figure 2. Apparent brightness of specular and matte surfaces at near and far positions, under (A) high illumination, and (B) low illumination.

matically matched pair of surfaces would appear brighter when both were under the same illumination. What actually happened was that specular surfaces appeared brighter than matte surfaces regardless of the achromatic colors of the surfaces. The dark-gray specular cylinder, for example, appeared brighter than the white matte cylinder. This result was general in that it occurred for high and low illumination and for objects at different distances from the observers.

Independence. To demonstrate independence between apparent brightness and achromatic color, one would want to show that a specular TF appears brighter than a matte TF but does not at the same time appear lighter in color. Were lightness and brightness to be correlated, that would diminish the argument for independence. What happened in much of the data, as indicated just above, is that the specular TFs appeared brighter even though they appeared darker, not lighter, than the matte TFs. To make this matter quite explicit, consider the frequency counts in Table 1.

To start, note the lop-sided frequencies in Rows 1 and 2 of the table. In Row 1, for example, the frequency of 212-10-6 means that in 212 of the 228 comparisons for 38 subjects the dark-gray specular TF appeared darker in color than all matte TFs of higher diffuse reflectances (viz., than the middle-gray, light-gray, and white matte TFs). There were 10 reversals and 6 ties. Correspondingly, also in Row 1, in 304 of 304 comparisons and in 76 of 76 comparisons, the black and light-gray specular TFs, respectively, appeared darker in color than matte TFs with higher diffuse reflectances, with no reversals or ties. These frequencies are, of course, precisely what one would expect of achromatically different TFs.
What one might not expect, however, are the frequencies in Row 2 of the table. Those frequencies refer to brightness comparisons, for a different group of 38 subjects. (The frequencies for the remaining 8 of the 46 brightness subjects are given in Row 3 of the table.) The frequency of 215-12-1 in Row 2, for example, means that in 215 of the 228 comparisons, the dark-gray specular TF appeared brighter than all matte TFs with higher diffuse reflectances (viz., than the middle-gray, light-gray, and white matte TFs), and that there were 12 reversals and 1 tie. The frequencies in Row 2 for the brightness judgments were composed, therefore, exactly as were the frequencies in Row 1 for the achromatic color judgments. (The only comparisons that could logically be allowed in Rows 1 and 2 involved specular surfaces that had lower diffuse reflectances than the matte surfaces with which they were compared. Otherwise, if the specular TF had a higher diffuse reflectance than the matte TF, its lighter achromatic color and its greater brightness would be correlated. For each of the 38 subjects in the first and sec-

Table 1

Frequencies from Experiment 1 Showing Specular Surfaces (TFs) Appeared Brighter But Darker Than Matte Surfaces (TFs) That Had Higher Diffuse Reflectances

\begin{tabular}{ccccc} 
No. of & \multicolumn{4}{c}{ Specular TFs } \\
\cline { 2 - 5 } Subjects & Black & Dark Gray & Middle Gray & Light Gray \\
\hline \multicolumn{5}{c}{ Achromatic Comparisons } \\
38 & $304-0-0$ & $212-10-6$ & $134-13-5$ & $76-0-0$ \\
38 & $302-1-1$ & $215-12-1$ & $134-14-4$ & $61-12-3$ \\
8 & $15-45-4$ & $2-12-2$ & $10-35-3$ & $7-23-2$ \\
\hline
\end{tabular}


ond rows of the table, there were $8,6,4$, and 2 allowable comparisons, respectively, for the four specular TFs from black to light gray.)

For the 76 subjects in Rows 1 and 2, the evidence is overwhelming. The specular TFs appeared brighter but darker than the matte TFs. In these comparisons, the specular TFs had lower diffuse reflectances and, presumably, lower overall average luminances than the matte TFs with which they were compared; and they also appeared darker. (See section below on overall average luminances.)

The eight subjects in Row 3 of the table are different. These 8 subjects, 4 each from the low and high illumination conditions, were those with the greatest numbers of reversals. Their results are interesting. They are in almost perfect disagreement with the other 38 subjects. For the 8 subjects, the matte TFs appeared brighter than the specular TFs in most comparisons-a near-perfect reversal. We have no explanation for the results of those 8 subjects, who comprised $17 \%$ of the total group of 46 brightnesscomparison subjects. (The 8 subjects serve as a reminder of the danger of small $\mathrm{Ns}$, particularly $\mathrm{Ns}$ of 1 or 2 " $\mathrm{ex}$ pert" observers. With an $N$ of 10 , for example, one might, by chance, draw a group like the 8 subjects, with their nontypical pattern of reversed responses.)

Overall average luminances. In assessing the specific luminance determinants for the brightness comparisons of the 38 subjects in Table 1 , we integrated luminance measures taken from Figure 1 over small areal regions of the test objects. In each case, the achromatically lighter matte objects had higher overall (arithmetic and, alternatively, logarithmic) average luminances than the achromatically darker specular objects with which they were compared in Table 1. We concluded from this that it was the intensity of specular luminances (or their apparent intensities), not overall average luminances, which determined apparent brightness, under our conditions in Experiment 1. Even this conclusion, however, is subject to

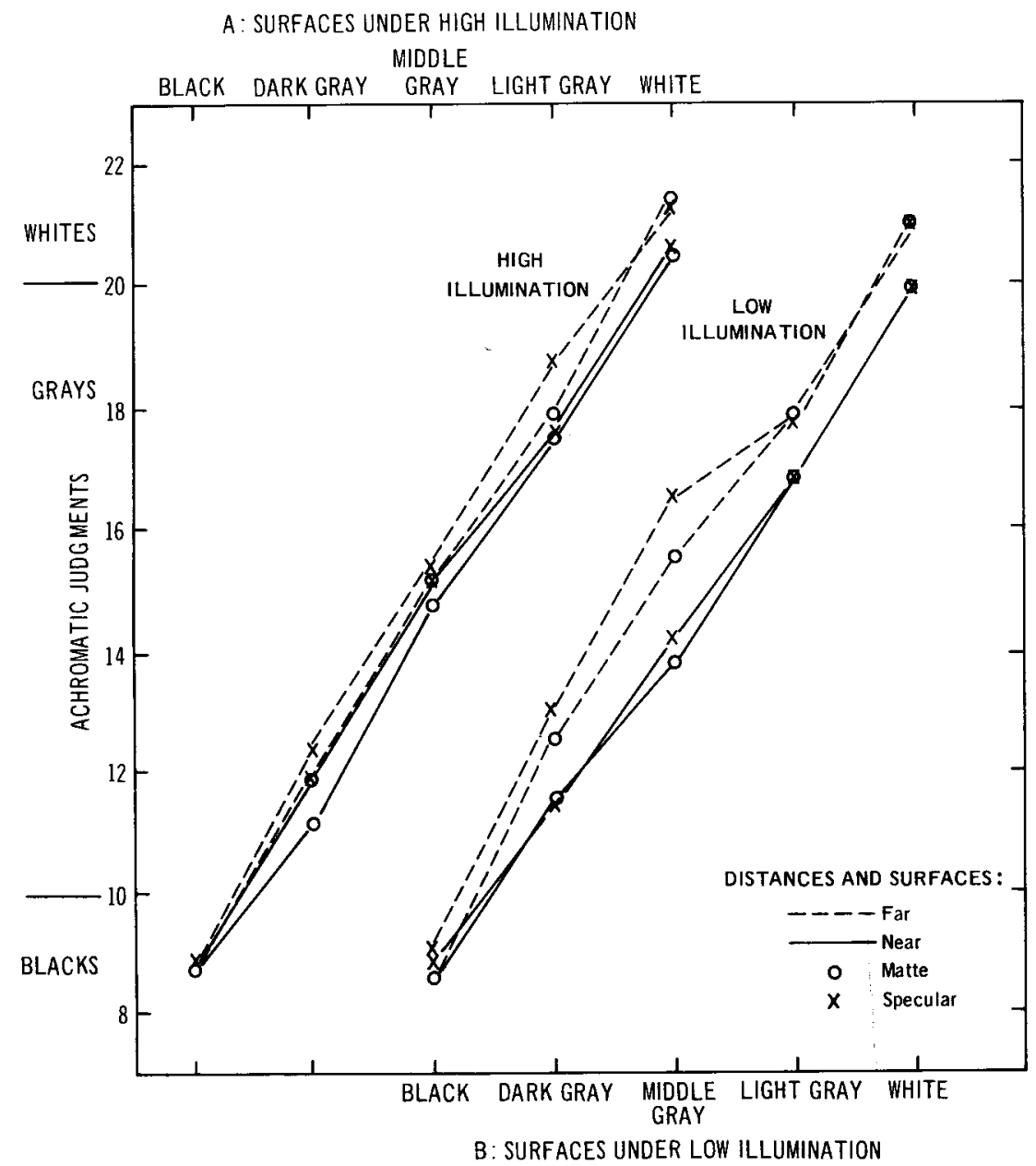

Figure 3. Apparent achromatic color of specular and matte surfaces at near and far positions, under (A) high illumination, and (B) low illumination. 
the caveat, articulated earlier, with respect to the danger of imposing on visual processors our arithmetic conceptions of how to average luminance intensities.

Shapes of achromatic functions. The achromatic color results are represented in Figure 3, where the means for the achromatic color judgments are plotted. On the left and right of the figure, the two bundles of four lines are for high- and low-illumination, respectively. The five TFs from black to white are shown on the two abscissas. The abscissa for high illumination is above the graph; the one for low illumination is arbitrarily displaced to the right and is below the graph. This displacement allowed for the separation of the two bundles. (Otherwise, with each group's achromatic judgments spreading over the entire number-scale, from 8 to 22 , the bundles would have been superimposed.)

The shapes of the four achromatic color functions in Figure 3 are approximately linear, and they rise steeply in a positive direction from lower left to upper right. It is, then, approximately true that as TFs were varied from black to white, apparent achromatic color shifted in comparable steps, from black to white. This occurred irrespective of whether the TFs were matte, specular, near, or far, or were under high or low illumination. The steeply rising, positively monotonic functions in Figure 3 produced large significant effects in two ANOVAs. The evaluations for the main effects of achromatic color from black to white yielded $F(4,108)=648, N=28$, for high illumination and $F(4,36)=97, N=10$, for low illumination. (Because of the very large $F$ of $648, N$ was reduced from 28 for high illumination to 10 for low illumination.)

One other significant statistical effect occurred in the data represented by Figure 3. Closer TFs were slightly, but reliably, darker than farther TFs. This main effect for distance can be seen in the figure, where solid lines for near objects were below the dashed lines for far objects $[F(1,27)=11, p<.005$, for high illumination; $F(1,9)=23, p<.001$, for low illumination]. This effect was expected, as indicated in the Apparatus section, because of the different luminance relations found between the TF and its background, when the TF is far and when it is near. It is cited here simply to emphasize how determinant luminance ratios are in specifying apparent achromatic color.

One nonsignificant effect in Figure 3 is relevant. Paired matte and specular TFs appeared approximately the same color. This occurred when the TFs were viewed near (pairs of dashed lines) and far (pairs of solid lines), under high and low illumination. In the two ANOVAs, the evaluations for the main effects of matte and specular surfaces yielded $F(1,27)=2.82$ for high illumination, and $F(1,9)=2.38$ for low illumination $(p s>.10$ ). Also, the six $F$ s for interactions between matte and specular surfaces, on the one hand, and other variables were of even less statistical consequence, varying from 0.50 to 1.03 .

Shapes of brightness functions. The shapes of the brightness functions in Figure 2 differed dramatically from the achromatic functions in Figure 3 . The functions in Figure 3 are steeply rising positive functions, whereas those in Figure 2 appear to have very different shapes. The four functions in the lower halves of Figure 2 for the matte surfaces appear to have some flat segments and some slightly rising segments. In comparison, the four functions in the upper halves of Figure 2, for specular surfaces, appear, overall, to be negative, decreasing functions. If these visual impressions are more or less (statistically) reliable, then the shapes of the brightness functions in Figure 2 and those of the achromatic functions in Figure 3 are not correlated functions. That would be further evidence for the independence of achromatic color and its brightness.

Consider first the four "brightness lines" for matte surfaces in the lower half of Figure 2. None of the rising segments in those lines was statistically significant. A separate rank-order Friedman chi-square was calculated for each of the four lines. The four chi-squares, each with $4 d f$, varied from 0.90 to 4.25 , with no $p$ smaller than .40 . The conclusion, given such small chi-squares, was that the four brightness functions (far, near, high, and low illumination) for the matte surfaces were not rising functions. Other data from the preliminary training (see Procedure section) supported the same conclusion. During training, the 46 "brightness" subjects observed the 10 TFs together and judged which was least bright. Their responses were distributed almost perfectly uniformly over the five matte TFs. No specular TF was chosen.

The finding that matte surfaces did not produce a rising brightness function is not unique, as indicated in the introduction to this paper.

Consider next the shapes of the four "brightness lines" for the specular surfaces in the upper halves of Figure 2. They appear to be negative decreasing functions. In each half of the figure, the pair of specular brightness lines converge on the pair of matte brightness lines, reading from left to right. This implies that there was a significant statistical interaction for each half of the figure. The interactions of surface (matte to specular) $\times$ color (the five surfaces from black to white) were, in fact, significant $[F(4,108)=15$ and $F(4,68)=6.52$, for high and low illumination, respectively; $p s<.001$ ]. This established what can be seen in Figures $2 \mathrm{~A}$ and $2 \mathrm{~B}$ : the matte and specular brightness functions converge on each other and thus have different shapes.

These analyses demonstrate what was claimed earlier. The shapes of the brightness and achromatic functions in Figures 2 and 3, respectively, are not correlated. That fact supports the contention that achromatic color and its brightness are independent phenomena.

Shapes of specular brightness functions. The specular brightness functions in the upper halves of Figure 2 appear to be either L-shaped or U-shaped. Although the topic of the shapes of these functions is peripheral to this paper, the shapes are of interest in themselves. In the vision literature there is little to indicate the shape of specular brightness functions for achromatically different TFs.

As a first step in analyzing the shapes of these functions, four Friedman chi-squares were calculated for the specular surfaces, each with $4 d f$. The four chi-squares 
varied from 19.71 to 25.51 , and all $p$ s were much less than .001 . Most of the magnitude of these chi-squares had to be attributed to the black specular surfaces. For example, the black specular TF under high illumination and in the near position appeared brighter than its closest neighbor, the dark-gray specular TF with a frequency of 20-6 and 2 ties $(p<.01)$. For the same comparison under low illumination, the frequency was $13-4$, with one tie $(p<.03)$. For other comparisons involving the black specular TF the frequencies were even more extreme.

The magnitude of the effect of the black specular TF is the basis for concluding that the brightness function for the specular surface was at least L-shaped. However, when the black specular TF was eliminated from the four Friedman chi-squares, the chi-squares, although greatly reduced in magnitude, were still not at the level of random variation [for the far position, $\chi^{2} s(3)=12.03$, $p<.01$, and 8.02, $p<.05$, for high and low illumination, respectively; for the near position, $\chi^{2} s(3)=4.15$, $p<.25$, and 5.22, $p<.15$, for high and low illumination, respectively].

What remained as nonrandom variation in the chisquares, after the exclusion of the black specular TF, was a systematic contribution from the white specular TF. A further example of this contribution is to be found in the preliminary training data. When the 46 subjects were asked which surface appeared brightest, they most frequently chose the specular black TF $(n=28)$; but they also chose the white specular TF $(n=14)$, with four scattered responses. The black and white specular surfaces appeared brighter than other surfaces.

These results thus imply that the specular brightness functions were at least L-shaped but more likely U-shaped.

\section{Experiment 2}

The results of Experiment 1 supported the two main hypotheses set forth in this paper. First, specular surfaces appeared brighter when under the same illumination as matte surfaces. Second, the greater brightness found for the specular surfaces than for the matte surfaces was independent of any differences in achromatic color between the matte and specular surfaces. That suggests that apparent achromatic color and brightness are independent orthogonal phenomena. The purpose of Experiment 2 was to replicate those findings under somewhat different conditions and with different procedures from those used in Experiment 1. Briefly, the conditions were arranged so that specular objects would mirror primary, instead of secondary, light sources. In another variation, sets of textured wood objects were painted to be either specular or matte. Sets of objects were presented in small rooms made of plywood and painted matte black. The room walls were undifferentiated, low-reflecting, low-luminance surfaces. Only to a negligible extent might those black walls illuminate the specular objects. Also, whatever image of them was formed on the specular surfaces would markedly differ from the specular images formed in Experiment 1.
Viewing was wide-angle and binocular, rather than monocular, and it included the floor and three walls.

The procedures for judging achromatic colors and brightness also differed. In this experiment, the same subject made both judgments. In addition, instead of scaling achromatic color and brightness, as in Experiment 1, the subject named the achromatic color of the matched pair of matte and specular test objects and directly compared their brightnesses.

\section{Method}

Subjects. The 40 subjects were recruited as were those in Experiment 1 . They had the same characteristics but, just prior to this experiment, had participated in a different experiment.

Apparatus. Two identical small rooms, each $86.3 \mathrm{~cm}$ wide, $122 \mathrm{~cm}$ high, and $168.5 \mathrm{~cm}$ deep, were constructed out of plywood and painted matte black (Munsell N2.3). Each was viewed binocularly through a viewing hood in the front wall that allowed a view of roughly $35 \mathrm{cu} f \mathrm{ft}$ of the space. Six 15 -W bulbs, spread over the ceiling, and one 15-W bulb on the back side of the viewing hood illuminated each room. (A detailed description of the rooms and locations of bulbs is given on pp. 116-118 and in Figure 1 of Flock \& Nusinowitz, 1984.)

Test objects. There were eight sets of wooden objects. Each set was composed of two salad bowls ( $27 \mathrm{~cm}$ in diameter, $9 \mathrm{~cm}$ high), a salad spoon $(20 \mathrm{~cm}$ long), and an egg cup $(4 \mathrm{~cm}$ in diameter and $6 \mathrm{~cm}$ high). Each of four of the sets was painted a different neutral matte color: black (Munsell 2.3), dark gray (Munsell 3.9), light gray (Munsell 6.3), and white (Munsell 9.2). The four other sets were painted with neutral specular paint, so as to match within \pm 0.1 Munsell steps the achromatic colors of the matte sets. This produced four pairs of matched sets of objects.

A set of matte objects was spread out on the floor in the farther part of one of the matte black rooms. The matching set of specular objects was placed in the same positions in the second room. The many images on the surfaces of the specular test objects were nonrepresentational highlights that directly mirrored the primary light sources in the ceiling or their reflections from other surfaces.

Maximal luminances. Maximal luminances in foot-lamberts of the specular and matte pairs of matched test objects, respectively, were: black, 28 and 0.29 ; dark gray, 12 and 0.52 ; light gray, 40 and 1.8; and white, 60 and 6.2. The maximal luminances in footlamberts for each wall were: floor, 0.24 ; far wall, 0.31 ; left wall, 0.19 ; and right wall, 0.22 . (The luminances differed from those reported in Table 1 of Flock \& Nusinowitz, 1984, because the equipment had been rebuilt and repainted and the socket locations had been slightly altered.)

Procedure. Four groups of 10 subjects each were assigned to conditions in which pairs of matched sets of objects were placed in the two black matte rooms-the matte objects in one room and the specular objects in the second room. For Group I, the objects were black; for Group II, they were dark gray; for Group III, light gray; and for Group IV, white.

The procedure was as follows. Subjects from Group III, for example, looked into one of the black matte rooms and named the achromatic color of the light-gray matte objects. (The subjects had to use the following color labels: very white, white, poor white, very light gray, light gray, middle gray, dark gray, very dark gray, poor black, black, very black.) They next looked at the light-gray specular objects (i.e., the matching set of the pair) in the second black matte room and named the colors of those objects. They were rerouted through the sequence and told to notice how bright the objects appeared. They were then asked, "Which set of objects appears brighter?" (The words matte and specular were never used.)Whether matte objects came first or second was counter- 
balanced. Otherwise, that judgmental order was the same for all subjects. There was an interstimulus interval during which the brightness comparisons were made. To view the second set of objects and make a brightness comparison, the subject had to move about $4 \mathrm{ft}$ in the darkness of the experimental space, mount a platform, and then look into the second chamber.

\section{Results and Discussion}

Despite the various changes in conditions, specular objects appeared brighter than the achromatically matched matte objects, just as they had in Experiment 1. Also, the paired matte and specular objects appeared the same color. These results implied orthogonality, as in Experiment 1.

Achromatic colors of the objects. The 10 subjects in each group judged the achromatic colors of the achromatically matched matte and specular objects. There were four sets of these repeated measures, a total of 40 pairs of achromatic measures. Over these 40 pairs of judgments, there were 21 ties, 10 judgments that the matte objects were lighter in color than the specular objects, and 9 judgments that the matte objects were darker in color than the specular objects. These frequencies supported the reliability of the prior matching of the achromatic pairs at nonspecular angles. The frequencies occurred randomly over the four groups and therefore over the four different achromatic colors of the objects.

Brightness of the objects. The specular objects appeared brighter than the phenomenally matched matte objects to 32 of the 40 subjects. The 8 exceptions occurred when the matte objects were presented first, the specular objects second. This suggested a primacy effect. On the 20 occasions when specular objects came first in the sequence, they appeared brighter with a frequency of 200 . When they appeared second in the sequence, they appeared brighter than matte objects with a frequency of 12 to 8 , thus overcoming the primacy effect. The eight exceptions occurred randomly over the four sets of achromatic colors.

Under the conditions of these experiments specular objects appeared brighter than phenomenally matched matte objects, again implying the independence of apparent brightness and achromatic color.

\section{Experiment 3}

In Experiments 1 and 2, specularity was varied over only two levels. In Experiment 3, it was varied over four levels, from matte to moderately high specular. The intent was to demonstrate that apparent brightness increases not only as a TF is varied from matte to specular, but also as it is varied over several levels of specularity.

\section{Method}

Subjects. Four groups, each with 24 subjects, were randomly assigned to the conditions described below. The 96 subjects were recruited as in Experiment 1, had the same characteristics, and had $20 / 20$ corrected or uncorrected vision in both eyes.

Test fields. Four black TFs were prepared. One was matte; three were specular, arbitrarily labeled as low, medium, and high. The four TFs were matched in color to a Munsell N2.3, \pm 0.1 (poor black in color). The four TFs were presented in all possible pairings. The observer chose which of each pair appeared brighter. This experiment was then replicated exactly, using light-gray TFs, matched to a Munsell N6.3. All TFs were upside-down wooden bowls, $27 \mathrm{~cm}$ in diameter.

The black achromatic TFs were produced using appropriate mixtures of Glidden interior-exterior enamel paints. To get a match of the paints required a lengthy trial-and-error process, using the following paints: No. 908 flat black (for the matte), No. 4620 semigloss black (for the low specular), No. 909 gloss black (for the medium specular), and No. 909 gloss black plus two coats of Elethane polyurethane plastic finish (No. 300) gloss (for the high specular). In addition, the colors of the specular black paints had to be adjusted by adding various amounts of No. 900 gloss white, to produce the medium and high specular colors, and No. 4700 semigloss white, to produce the low specular color. Also, small amounts of Dramatone colorants, No. 1768 oxide red and No. 1752 oxide yellow, were added to neutralize blue tints in the paint. The four light-gray TFs were similarly produced, using Glidden's No. 7949 paints. Specifically, these were interior alkyd flat, No. 5787 (matte); interior alkyd semi-gloss, No. 4687 (low specular); interior-exterior enamel, No. 987 (medium specular); and No. 987 plus Liquitex acrylic gloss medium, No. 5008 (high specular). Also, small amounts of colorants were used: Dramatone No. 1768 oxide red, in the matte; No. 1782 black and No. 1750 neutral toner, in the low specular; and No. 1757 violet, in the medium and high specular.

Displays. Three small black matte rooms were used. These were modified versions of those used in Experiment 2. A vertical partition, $122 \mathrm{~cm}$ high $\times 30 \mathrm{~cm}$ deep, was placed in the back of each room, subdividing the rear of the room into two adjacent spaces. Each of a pair of bowls, placed on the floor in the subdivided spaces, was illuminated by a separate GE 75-W Shadow Ban incandescent bulb, placed directly over it. The light from these bulbs also illuminated the remainder of the space.

All possible pairings of the four levels of black bowls (one matte, three specular) would have required six small rooms. Only three were available. Therefore, two separate groups of subjects were used for the six comparisons. Group I observed all possible pairings of the three specular black bowls. Group II observed the black matte bowl paired with each of the three specular bowls. Groups III and IV observed light-gray bowls, in an exact replication of the conditions for Groups I and II.

Procedure. In the paired comparisons, a subject judged which TF appeared brighter. For Group I, for example, the three comparisons involved the specular black TFs, paired as follows: high and low, high and medium, medium and low. Each subject viewed the three rooms in a different counterbalanced order. The sequence was repeated two more times, in different orders. In these two additional replications, the subject judged which TF appeared "glarier" and "glossier," in that order. The purpose of these two additional replications for each subject was to examine the validity of the experiments-whether, for example, the use of the term brightness was independent of the use of the terms glary and glossy. The results of these validity studies are presented separately in a later section.

\section{Results}

Each subject made three comparison judgments for brightness, making for a total of 288 comparisons over the four groups of subjects. In 261 of these 288 comparisons, or in $91 \%$ of them, the subjects reported that the more specular of a pair of surfaces appeared brighter. This result gave overwhelming support to the earlier findings 
in Experiments 1 and 2 that as specularity is increased, apparent brightness correspondingly increases. (The frequencies for this experiment are given in Table 3.)

\section{Experiment 4}

In Experiments 1 to 3, illumination was always the same for all TFs. In Experiment 4, identical specular TFs were placed under two different illuminations, one under a 100 $\mathrm{W}$ bulb and the other under a $40-\mathrm{W}$ bulb, producing an illumination ratio of 2.2:1. The TFs were the three pairs of black specular bowls used in Experiment 3. All other conditions were the same as in Experiment 3, including judgments of which TF appeared glarier and which appeared glossier. A new group of 24 subjects was recruited for this experiment, with characteristics the same as before.

The specular surface under the higher illumination, with its higher maximal luminance, was reported to appear brighter than its identical match under lower illumination. The frequency was 70-2. Had degree of specularity alone determined the result, the expected frequencies would have been 36-36. Hence, higher maximal luminances, rather than a particular level of specularity, seemed to determine apparent brightness.

In this experiment, the brighter of the two matched surfaces was more highly illuminated. The higher diffuse reflections that resulted might have contributed, at least in part, to the greater brightness of the surface. That possibility was investigated in Experiment 5.

\section{Experiment 5}

The focus of Experiments 1 to 4 was on specular reflections as stimuli for the apparent brightness of surfaces. As explained in the introduction, specular highlights are a major class of visual stimuli, but by no means the only class that can account for a surface's apparent brightness. In the absence of specular highlights, for example, matte surfaces do appear bright. The results of Experiment 4 could be interpreted to mean that the more highly illuminated surface appeared brighter not only because it had more intense highlights, but also because it and its surrounds had higher diffuse luminances. Were that the case, then the diffuse luminances of specular surfaces and their surrounds might also contribute to their apparent brightness.

One way to demonstrate this would be to show that a less specular surface under high illumination, with high diffuse luminances, can appear brighter than a more specular surface that is under low illumination. In Experiment 5 , then, the illumination was arranged so that the more specular surface (under lower illumination) possessed the highest specular intensities, but the less specular surface and its surrounds (under higher illumination) possessed higher diffuse luminances. Otherwise, everything in the two scenes was equal.

\section{Method}

Subjects. Ninety-six new subjects were recruited as in Experiment 3, and had the same characteristics.

Luminances, displays, and procedures. The one matte and the three specular black bowls (matched to a Munsell N2.3) that had been used in Experiment 3 were the TF objects. Pairs of these four bowls were simultaneously presented and compared, with each of the pair under different illumination. In each comparison, the more specular bowl was under lower illumination than the less specular bowl. Ilumination was controlled by placing a $100-\mathrm{W}$ bulb over the less specular bowl and a $40-\mathrm{W}$ bulb plus appropriate neutral density filters over the more specular bowl. (The bulbs were GE Shadow Bans.)

Except for these changes in illumination, all displays, conditions, and procedures in Experiment 5 were the same as those in Experiment 3 , as were the responses required of the subjects.

The illumination conditions for this experiment are described in Table 2. Each section in the table gives relevant information about luminance conditions and the results for Groups I-IV, each of which had 24 different subjects. In explanation, consider the three pairs of rows of luminances used for Group 1. Each pair of rows corresponds to a comparison between a more and a less specular bowl (TF). For each of the three comparisons, luminance measures are given in foot-lamberts, that describe maximal (Max) and minimal (Min) luminances for the specular reflections and the diffuse reflections for each of the two compared bowls. Thus, each subject in Group I made three comparison judgments, reporting for each comparison which of the two bowls appeared brighter. For the comparison involving Rows 1 and 2, notice in Row 1 that the Max and Min specular luminances of the more specular bowl, under lower illumination, were 52 and 40 . Those specular luminances were higher than the specular luminances of 18 and 14, respectively, in Row 2, for the more highly illuminated but less specular bowl. In comparison, the Max and Min diffuse luminances of the more specular bowl were lower, .045 and .030 than the diffuse luminances for the less specular bowl, .27 and .19 , respectively. The ratio of the maximal specular luminances, 52 to 18 , or 2.9 to 1 , is given between the two rows.

The comparison frequency for the 24 subjects is given in the far right column. Although results are discussed below, notice that in this comparison, the more specular surface appeared brighter to only 6 of the 24 subjects, whereas the less specular surface appeared brighter to 18 of the 24 subjects (binomial probability, $p<.01$ ).

For Groups III and IV, specular luminances could not be identified on the matte bowls. There were, however, regions of higher and lower luminances on the matte bowls, each with a maximum and minimum. Those maxima and minima are given in the table.

\section{Results}

The results for each comparison are given, as already indicated, in the form of frequencies in the far column of Table 2. The first and second numbers in each frequency entry are the number of times in a comparison that the more specular surface appeared brighter or less bright, respectively, to the group of 24 subjects. The frequencies within each section are within effects, whereas comparisons between sections are between effects.

The frequencies indicate that a more specular surface no longer necessarily appeared brighter as it had in Experiments 1 to 3 . For Group I, for example, the summed frequencies show that the more specular bowls appeared brighter in only 13 , or $18 \%$, of the 72 comparisons. For 
Table 2

Frequencies Showing the Number of Times in Three Comparisons (Each Pair of Rows Specifying a Comparison) Four Groups ( $n s=24)$ Chose the More Specular Surface as Brighter (First Number in a Frequency) Versus Less Bright (Second Number in a Frequency) in Experiment 5

\begin{tabular}{|c|c|c|c|c|c|c|c|}
\hline \multirow[b]{2}{*}{ Group } & \multirow[b]{2}{*}{ Condition } & \multicolumn{2}{|c|}{$\begin{array}{c}\text { Specular } \\
\text { Luminance }\end{array}$} & \multicolumn{2}{|c|}{$\begin{array}{c}\text { Diffuse } \\
\text { Luminance }\end{array}$} & \multirow{2}{*}{$\begin{array}{c}\text { Ratio of } \\
\text { Maximal } \\
\text { Specular } \\
\text { Luminances } \\
\end{array}$} & \multirow[b]{2}{*}{ Frequency } \\
\hline & & $\operatorname{Max}$ & Min & $\operatorname{Max}$ & Min & & \\
\hline \multirow[t]{4}{*}{$\mathbf{I}$} & $\begin{array}{l}\text { High Specular TF } \\
\text { Low Specular TF }\end{array}$ & $\begin{array}{l}52 \\
18\end{array}$ & $\begin{array}{l}40 \\
14\end{array}$ & $\begin{array}{l}.045 \\
.27\end{array}$ & $\begin{array}{l}.030 \\
.19\end{array}$ & 2.9 & 6-18 \\
\hline & $\begin{array}{l}\text { High Specular TF } \\
\text { Medium Specular TF }\end{array}$ & $\begin{array}{l}52 \\
29\end{array}$ & $\begin{array}{l}40 \\
20\end{array}$ & $\begin{array}{l}.045 \\
.09\end{array}$ & $\begin{array}{l}.03 \\
.071\end{array}$ & 1.8 & $4-20$ \\
\hline & $\begin{array}{l}\text { Medium Specular TF } \\
\text { Low Specular TF }\end{array}$ & $\begin{array}{l}34 \\
18\end{array}$ & $\begin{array}{l}17 \\
14\end{array}$ & $\begin{array}{l}.075 \\
.27\end{array}$ & $\begin{array}{l}.062 \\
.19\end{array}$ & 1.9 & $3-21$ \\
\hline & & & & & \multicolumn{2}{|c|}{ Summed Frequencies } & $13-59$ \\
\hline \multirow[t]{5}{*}{ II } & $\begin{array}{l}\text { High Specular TF } \\
\text { Low Specular TF }\end{array}$ & $\begin{array}{l}95 \\
18\end{array}$ & $\begin{array}{l}70 \\
14\end{array}$ & $\begin{array}{l}.062 \\
.27\end{array}$ & $\begin{array}{l}.048 \\
.19\end{array}$ & 5.3 & $10-14$ \\
\hline & $\begin{array}{l}\text { High Specular TF } \\
\text { Medium Specular TF }\end{array}$ & $\begin{array}{l}95 \\
29\end{array}$ & $\begin{array}{l}70 \\
20\end{array}$ & $\begin{array}{l}.062 \\
.09\end{array}$ & $\begin{array}{l}.048 \\
.07\end{array}$ & 3.3 & $11-13$ \\
\hline & $\begin{array}{l}\text { Medium Specular TF } \\
\text { Low Specular TF }\end{array}$ & $\begin{array}{l}49 \\
18\end{array}$ & $\begin{array}{l}28 \\
14\end{array}$ & $\begin{array}{l}.10 \\
.27\end{array}$ & $\begin{array}{l}.09 \\
.19\end{array}$ & 2.7 & $9-15$ \\
\hline & & & & & \multicolumn{2}{|c|}{ Summed Frequencies } & $30-42$ \\
\hline & & & Tota & ecular & isons & oups $I$ and II) & 43-101 \\
\hline
\end{tabular}

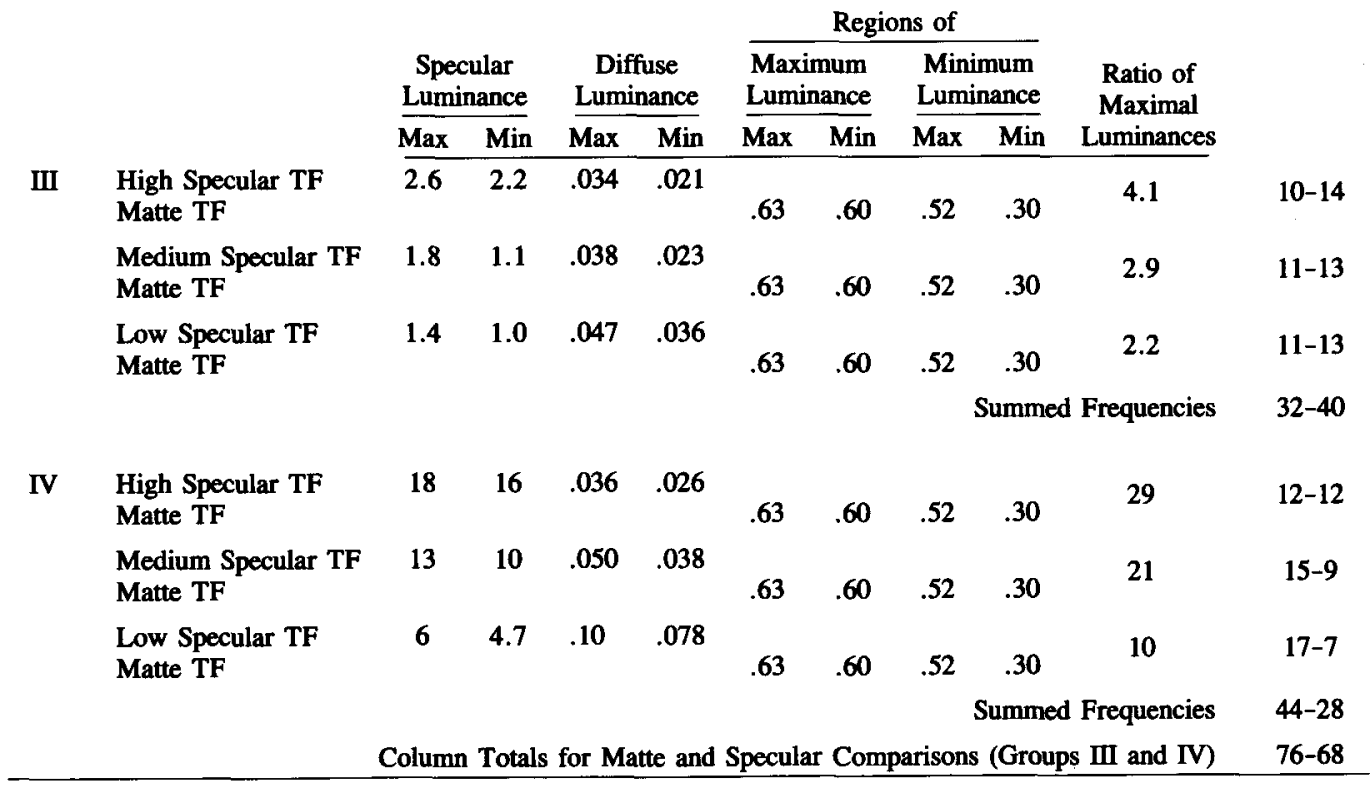

Note-The more specular surface is always under lower overall illumination. Maximal and minimal luminances in footlamberts are given for the specular and diffuse luminances of each surface (TF) in a comparison. All TFs matched to a Munsell N2.3 black.

those luminance arrangements, the greater intensity and larger areal extents of the diffuse reflections of the less specular surface and its surrounds seemed to determine the relative brightness of the two bowls. Higher maximal luminances and a higher level of specularity of the more specular surface played little role under low illumination.

From Group I to Group II, the luminances of the more highly illuminated but less specular bowls were kept constant, whereas the luminances of the more specular bowls (and their surrounds) were increased. (For example, compare the luminances given in Rows 1 and 2 for Groups I and II.) The response frequencies correspondingly increased. For Group II, the more specular bowls appeared brighter in 30 , or $42 \%$, of the 72 comparisons, which was a marked shift from the $18 \%$ for Group I. Under these changed luminance conditions, the more specular TF appeared to be, on average, nearly as bright as the less specular TF. It was as if an approximate balance existed 
between the more intense specular highlights in the one scene under lower illumination and the higher diffuse luminances in the other scene under higher illumination.

The frequencies for Group III were similar to those for Group II. The more specular surface appeared brighter in 32 , or $44 \%$, of the 72 comparisons.

From Group III to Group IV there is again a marked change in the frequencies. For Group IV, the frequencies were $44-28$, or $61 \%$. [The chi-square for the four sets of frequencies, each set divided by 3 to produce independence, was 9.34(3), $p<.03$.] Only for Group IV did the more specular surfaces appear brighter, on average, than the less specular surface. (The binomial probability of the $15-9$ split, dividing $44-28$ by 3 , was $<.20$.) This change in response is presumed to have occurred because of the changes in specular luminances from Group III to Group IV. The luminances of the less specular surfaces were held constant, whereas those for the more specular surfaces were increased. (Note these changes in luminance, by comparing, for example, the luminances in the first rows for Groups III and IV.)

For Groups I and II and separately for Groups III and IV, luminances of the less specular surface were always held constant. That was not the case between Group I and Group IV (or between Group II and Group III). When we attempted to rationalize the large change in response between Group I and Group IV, by an analysis of the luminances, we were unable to discover the appropriate algorithms for the luminances to account for the response changes. We were unable to determine how to weight and configurate the diffuse and specular luminances so as to account for the response change from $18 \%$ for Group I to $61 \%$ for Group IV. [That statistically significant change gave $\chi^{2}(1)=7.6, p<.01$, including Yates's correction.] Correspondingly, we were unable to rationalize the absence of response changes between Groups II and III.

The relationships in Table 2 are manifestly complicated. Consider the two scenes in each of the three comparisons for Group IV. The less specular surfaces were under levels of illumination that were 6 to 15 times greater than those for the more specular surfaces. Despite that large difference in illumination, the more specular surfaces under lower illumination appeared brighter, on average. The determinant factor was surely their specular intensities (maximal luminances), which were from 10 to 30 times greater than those in the more highly illuminated scenes.

In comparison, consider Group I. The specular intensities of the more specular surfaces were approximately 2 to 3 times greater than the specular intensities for the less specular surfaces (versus magnitudes of 10 to 30 for Group IV). At that level of magnitude, the advantage in specular intensity was insufficient to overcome large differences in overall illumination. (The level of overall illumination of the less specular surfaces was 2 to 6 times greater.) This conclusion is indicated by the response frequency of 59-13 that the less specular surface appeared brighter. (The binomial probability, dividing $59-13$ by 3 , for a 19-5 split, is < .003.)
Table 3

Frequencies for Experiments 3 and 5 and Experiment 4

\begin{tabular}{|c|c|c|c|}
\hline & Brighter & Glossier & Glari \\
\hline \multicolumn{4}{|c|}{$\begin{array}{l}\text { A. Comparisons Among Matte and Specular TFs } \\
\text { Under Same Illumination (Experiment 3)* }\end{array}$} \\
\hline $\begin{array}{l}\text { Black TFs } \\
\text { Specular TFs Only } \\
\text { Matte and Specular TFs }\end{array}$ & $\begin{array}{l}71-1 \\
65-7\end{array}$ & $\begin{array}{l}67-5 \\
72-0\end{array}$ & $\begin{array}{r}55-17 \\
70-2\end{array}$ \\
\hline $\begin{array}{l}\text { Light-Gray TFs } \\
\text { Specular TFs Only } \\
\text { Matte and Specular TFs }\end{array}$ & $\begin{array}{c}62-10 \\
63-9\end{array}$ & $\begin{array}{l}63-9 \\
72-0\end{array}$ & $\begin{array}{l}67-5 \\
71-1\end{array}$ \\
\hline $\begin{array}{r}\text { Summed Frequencies } \\
\text { Proportions }\end{array}$ & $\begin{array}{c}261-27 \\
0.91\end{array}$ & $\begin{array}{c}274-14 \\
0.95\end{array}$ & $\begin{array}{r}263-2 \\
0.91\end{array}$ \\
\hline
\end{tabular}

B. Comparisons Among Matte and Specular TFs With the More Specular TF Under Lower Illumination

But With a Higher Maximal Luminance (Experiment 5)*

Black Specular TFs Only

$\begin{array}{rccc}\text { Group I } & 13-59 & 60-12 & 53-19 \\ \begin{array}{l}\text { Group II } \\ \text { Black Matte and Specular TFs }\end{array} & 30-42 & 65-7 & 47-25 \\ \text { Group III } & 32-40 & 70-2 & 70-2 \\ \text { Group IV } & 44-28 & 72-0 & 67-5 \\ \text { Summed Frequencies } & 119-169 & 267-21 & 237-51 \\ \text { Proportions } & 0.41 & 0.93 & 0.82\end{array}$

C. Frequencies Showing That of Two Identical TFs The One Under Higher Ilumination Appeared Brighter, Glossier, and Glarier (Experiment 4)

Black Specular TFs Only $\quad 71-1 \quad 48-24 \quad 63-9$

\begin{tabular}{llll} 
Proportions & 0.99 & 0.67 & 0.88 \\
\hline
\end{tabular}

Note-Each row of frequencies refers to a different group of 24 subjects. *The first number in each frequency entry indicates how frequently the more specular of two surfaces (TFs) appeared brighter, glossier, or glarier.

These comparisons are exemplary. Presumably, there is an algorithm to be discovered. We need to learn how the visual system, in determining the apparent brightness of surfaces and scenes, weights differences in intensity of the diffuse and specular reflections; we need to discover the role played by the areal extent of these differing luminances, and we need to know how the visual system averages complexly different luminance intensities.

\section{The Phenomenology of Apparent Brightness, Gloss, and Glare}

Early in the design of these experiments, we anticipated questions about the validity of the expected results. Under the conditions and demands of these experiments, observers might have been using the term brighter, when in fact objects appeared to them to be glossier or glarier. To explore this question, subjects in Experiments 3 to 5 were required to choose not only which of the paired TFs appeared brighter, but also which appeared glossier and glarier, as described in the Procedure section of Experiment 3.

The results of these comparison judgments are given in Table 3. There, for Experiment 3 (Table 3A), when matte and specular surfaces were under the same illumi- 
nation, the more specular surface did in fact appear brighter as well as glossier and glarier. The frequencies on which this conclusion depends are given in Table 3A as: $261-27,274-14$, and 263-25, respectively. For the conditions specific to Experiment 3, therefore, one might play the devil's advocate and conclude that the subjects were unable to discriminate "brightness" from glossiness and glare.

Consider the results, however, for Experiment 5, in Table 3B. The more specular surface appeared brighter in only 119 of the 288 comparisons (a proportion of .41 ), whereas it continued to appear overwhelmingly glossier and glarier (frequencies of 267-21, a proportion of .93, and 237-51, a proportion of .82, respectively). From these results, we can infer that an observer did in fact mean that a surface appeared bright, not glossy or glary, when it was reported to appear bright. Observers did, it seems, selectively discriminate brightness from glossiness and glare in these experiments.

This independence was f:rther confirmed in Table 4B, which gives within comparisons for observers in Experiment 5. There, the particular surface that appeared brighter to a subject appeared glossier in only 118 of 288 comparisons, a proportion of .41 , and appeared glarier in 138 of 288 comparisons, a proportion of .48 (Columns 1 and 2, respectively). Those proportions were approximately at chance levels, indicating that phenomenal brightness was discriminatively independent of gloss and glare.

\section{The Phenomenology of Gloss}

In the psychological literature, except possibly for Beck and Prazdny (1981) and Bixby (1926), there have been few empirical attempts to relate apparent gloss (glossiness) to antecedent conditions, although there has been theoretical speculation about the relationship (viz., Beck, 1972; Evans, 1948, 1974; Helmholtz, 1925; Hering, 1905-1911/1964; Katz, 1935, etc.).

In applied research, a somewhat different situation has prevailed. Specialized instruments have been in use to index specularity and levels of specular intensities for different materials, such as plastics, varnishes, papers, textiles, and so forth. In instances in which visual ratings of apparent gloss were obtained, they were found to be positively correlated to these physical indices (viz., Fourt, Howorth, \& Rutherford, 1954; Hunter, 1958; Hunter \& Lofland, 1956; Matsuta \& Kubota, 1981; Nimeroff, 1952; O'Donnell \& Billmeyer, 1986). What is reported next, therefore, merely confirms the findings of this applied research.

In Experiments 3 and 5, apparent gloss was positively correlated with a surface's level of specularity. In those experiments, the more specular surface tended always to appear glossier, as indicated by the frequencies of 27414 and 267-21 in Column 2 of Table 3 . In considering the implications of this correlation, it should be kept in mind that the most specular surfaces used in these experiments were only moderately specular. As mirrors, they only crudely imaged their surrounds. Had they been much better mirrors, the reflection plane would have become increasingly invisible. As that occurs, the correlation between specularity and gloss would presumably break down.

The relationship between apparent gloss and a surface's level of specularity was put to a different test in Experiment 4 . The compared surfaces were made specularly identical but were illuminated differently. Because of its greater number of visible highlights and the greater intensity of those highlights, the surface under higher illumination would mimic a higher level of specularity. There might then be a tendency for that surface to appear glossier. In Table 3C, for the 24 subjects in Experiment 4, the observed frequency was 48-24 (a proportion of .67). Of those 24 subjects, 17 reported that the surface under higher illumination appeared glossier on at least two of their three comparisons. (The binomial probability of that split of $17-7$ is $<.03$. For the 17 subjects, the overall frequency was 45-6.)

\section{Glare}

Apparent glare was found to be discriminatively different from apparent brightness (as indicated by the frequency of 138-150 in Table 4B). We were unable to show, however, whether it was discriminatively different from apparent gloss. (The frequencies in the last column of Ta-

Table 4

Frequencies for Experiments 3 and 5 and Experiment 4

\begin{tabular}{lccc}
\hline & $\begin{array}{c}\text { Brighter } \\
\text { Glossier }\end{array}$ & $\begin{array}{c}\text { Brighter } \\
\text { Glarier }\end{array}$ & $\begin{array}{c}\text { Glossier } \\
\text { Glarier }\end{array}$ \\
\hline \multicolumn{4}{c}{$\begin{array}{c}\text { A. Comparisons of Matte and Specular TFs } \\
\text { Under Same Illumination (Experiment 3)* }\end{array}$} \\
$\begin{array}{l}\text { Black TFs } \\
\text { Specular TFs Only } \\
\text { Matte and Specular TFs }\end{array}$ & $68-4$ & $54-18$ & $54-18$ \\
Light-Gray TFs & $65-7$ & $65-7$ & $70-2$ \\
Specular TFs Only & & & \\
Matte and Specular TFs & $59-13$ & $61-11$ & $60-12$ \\
Summed Frequencies & $255-33$ & $242-46$ & $255-33$ \\
Proportions & 0.89 & 0.84 & 0.89
\end{tabular}

B. Comparisons of Matte and Specular TFs

With Specular TF Under Lower Ilumination

But a Higher Maximal Luminance (Experiment 5)*

Black Specular TFs

$\begin{array}{rccc}\begin{array}{l}\text { Group I } \\ \text { Group II }\end{array} & 17-55 & 30-42 & 53-19 \\ \text { Black Matte and Specular TFs } & 27-45 & 35-37 & 42-30 \\ \text { Group III } & 30-42 & 30-42 & 72-0 \\ \text { Group IV } & 44-28 & 43-29 & 67-5 \\ \text { Summed Frequencies } & 118-170 & 138-150 & 234-54 \\ \text { Proportions } & 0.41 & 0.48 & 0.81\end{array}$

C. Comparisons of Identical Specular TFs

Under Different Illumination (Experiment 4)

$\begin{array}{cccc}\text { Matched Black Specular TFs } & 47-25 & 64-8 & 51-21 \\ \text { Proportions } & 0.65 & 0.89 & 0.71\end{array}$

\begin{tabular}{llll} 
Proportions & 0.65 & 0.89 & 0.71 \\
\hline
\end{tabular}

Note-Each row of frequencies refers to a different group of 24 subjects. *The first number in each frequency entry indicates how frequently the more specular of two surfaces (TFs) appeared both brighter and glossier, both brighter and glarier, and both glossier and glarier. 
bles $4 \mathrm{~A}$ and $4 \mathrm{~B}$ give proportions of .89 and .81 for glare and gloss. This implies that apparent glare, in this research, was correlated to a surface's level of specularity as well as to its apparent glossiness.)

\section{GENERAL DISCUSSION}

In Experiments 1 and 2, surfaces that were more and less specular were judged as to their apparent brightness and achromatic color. In these experiments, as specularity was increased, a surface appeared approximately the same achromatic color, but the more specular surface appeared brighter. Moreover, the more specular surface appeared brighter even when it was achromatically darker than the less specular surface and was also presumed to have a much lower average overall luminance. Finally, achromatic colors were varied phenomenally from black to white without a corresponding change in their apparent brightness (Figure 2).

The inescapable conclusion suggested by these experiments is that the apparent brightness of surfaces and their achromatic color are bidimensional and orthogonal. That was true for the various photopic levels of illumination that were used. Whether the dimensions would have been orthogonal for other levels of illumination is an empirical question to be determined only by future research.

Even in the absence of orthogonality for all levels of illumination, the current experiments have surely demonstrated the separateness of the two dimensions (viz., their bidimensionality). This issue of bidimensionality has been historically controversial. The claims for it date at least to Hering (1905-1911/1964), who reacted negatively toward Helmholtz's (1925) unidimensionality. Following Hering, numerous visual scientists (for example, those cited in the introduction as supporting luminance ratios, as well as other writers, such as Hsia, 1943; Katz, 1935; Lie, 1969; Noguchi \& Kozaki, 1985) have supported bidimensionality but not, necessarily, orthogonality. With the possible exception of Koffka (1932) and perhaps several other writers who implied orthogonality over limited ranges of illumination, most bidimensional theorists have claimed that the two dimensions were different but partially or wholly correlated. This expectation of a positive correlation between achromatic color and brightness may be the main reason why bidimensional theory has never had widespread support. Instead, according to Heggelund (1974), Helmholtz's unidimensional view dominated both theoretical thinking and empirical investigations in the visual sciences.

The unidimensional view is wrong, as the experiments in this paper demonstrate. The historical difficulty in demonstrating its wrongness may have occurred because of a number of conventions that have prevailed in the visual sciences. Chief among these has been the avoidance of specular surfaces. Invariably, experimental displays have been matte, flat, and untextured, and have been viewed monocularly with a small, often foveal, field of view by, in many instances, "trained" observers. Those have been the conventions. Such restrictive conditions reduced the degrees of freedom available to the observer. Because experimental conditions artifactually collapsed one of the more obvious brightness dimensions, that of specularity, the appropriate response variability disappeared. Orthogonality and bidimensionality could not then be easily demonstrated.

In response to the restrictive conventions that have prevailed, we increased the degrees of freedom associated with the displays and the viewing of them in our experiments. We used matte and specular objects, variously shaped and textured, presented in three-dimensional spaces. Observers were given a wide view of the scene, under both monocular and binocular conditions. Naive observers were used. The naive layman is an experienced observer of achromatic phenomena and a sophisticated user of visual language. He/she is naive only in terms of awareness of particular theoretical ideologies. As a result of our freer conditions and particularly the use of specular surfaces, it was easy to demonstrate that surfaces appeared more and less bright and that those appearances were orthogonal to the achromatic appearances of the surface.

The demonstration that surfaces could be made to appear brighter by increasing their specularity was shown, more generally, in Experiment 3. There, specularity was varied over four levels, from matte to moderately high specular. The apparent brightness of the specular surfaces correspondingly increased. Nevertheless, the determining variable in Experiments 1-3 was probably not specularity. With illumination constant in those experiments, the variations in levels of specularity were simply a device used to vary specular intensities, and, therefore, maximal (and perhaps even overall average) luminances. When pairs of the same specular objects were viewed under different levels of illumination in Experiment 4, the object under higher illumination, with higher specular intensities and maximal luminances, virtually without exception appeared brighter. It would seem, therefore, that judgments of brightness and relative brightness in Experiments 1-4 depended on which surfaces had the highest maximal luminances.

It is phenomenally self-evident that surfaces with no detectable specular highlights, such as matte surfaces, also appear bright. It should logically follow, therefore, that under appropriate conditions of illumination, less specular surfaces can be made to appear brighter than more specular surfaces. One aspect of that proposition was demonstrated in Experiment 5.

In Experiment 5, a more specular bowl in one scene was compared with a less specular bowl in a second scene. Except for their illumination, the two scenes were matched. The illumination of the scene with the less specular bowl was 2 to 6 times greater than the illumination of the second scene, but the specular intensities in the first scene were $1 / 2$ to $1 / 3$ those in the second scene. For those conditions, the less specular bowl under higher illumination (with higher diffuse luminances) appeared over- 
whelmingly brighter than the more specular bowl (with the highest maximal luminances) under lower illumination. Higher diffuse luminances and their areal extents, not higher maximal luminances, were presumed to be the determining factors.

This situation was reversed by making specular intensities for the more specular bowl 10 to 30 times greater than those for the less specular bowl while their intensity of illumination was $1 / 6$ to $1 / 10$ as great. Then, on average, the more specular bowl under much lower illumination appeared brighter.

In Experiment 5, therefore, it seemed that apparent brightness depended on some algorithm that involved maximum diffuse and specular luminances and their areal extents. In a particular scene that is filled with matte and specular surfaces that vary in achromatic colors and illumination, the surface that appears brightest will depend, presumably, on the output of that algorithm. Nevertheless, for simpler conditions, as in Experiments 1-4, the findings were, with few exceptions, straightforward. For pairs of surfaces with the same apparent achromatic color and the same (or, in Experiment 4, different) illuminances, the more specular surface (or the more intensely illuminated surface) invariably appeared brighter.

In this discussion we have not explicitly referred to the apparent intensity of illumination of a surface. Because the intensive highlights on surfaces are actual images of sources, the visual system is given an indirect, mirror "look" at the intensity of the illuminating sources, just about wherever it happens to gaze (Flock, 1984). As a result, these images or reflections of the sources might be a factor in determining not only the brightness of a surface, but also in how intensely it is being illuminated. This need not mean, however, that the two phenomena, intensity of illumination and brightness of surfaces, are the same, as, for example, Katz (1935) and Kozaki (1973) suggested.

In describing the appearance of the displays, the subjects in these experiments might have been saying "bright" when in fact they meant "glossy" or "glary." That was not the case, however. When that possibility was investigated in Experiments 3-5, it was found that subjects were distinguishing brightness from glossiness and glare.

Throughout this paper, we have assumed that the presence and apparent brightness of specular highlights increase the apparent brightness of the surrounding surface that embeds the highlights. (On page 441 above, this assumption was also extended to spatially discontinuous matte surfaces.) One might appropriately ask, over what areal extents do such effects prevail? The answer must await further research.

Also, we may have left the impression that all reasonably specular surfaces produce "focused" mirror images of the surrounding spaces. That is not always strictly true. Some substances (viz., lustrous textiles such as silks) reflect proportionately much specular light from their top (or first) surface and are, therefore, reasonably specular. Nevertheless, because of the roughness of their specular first surface, such first-surface light, analyzed over "large" areas, is found to be scattered light. This scattered light forms an averaged image of the ambient illumination, but it will not otherwise "mirror" the scene. This scattered light, therefore, is diffused light, but it carries information about the illuminating sources, not about the pigments and textures of the surface. Whether that first-surface diffuse light can be visually discriminated and separated from the diffuse light emanating from below the surface is a matter to be considered at a conceptual and theoretical level and would be a challenge as an empirical demonstration. What emerges here is a third variable, known to physicists but unacknowledged in the visual literature. We have yet to assess the separate perceptual effects of these three luminance variables: directional light from the first surface; scattered light from the first surface; and scattered light from below the first surface.

\section{REFERENCES}

BECK, J. (1964). The effect of gloss on perceived lightness. American Jourmal of Psychology, 58, 54-63.

BeCK, J. (1972). Surface color perception. Ithaca, New York: Cornell University Press.

Beck, J., \& Prazdny, S. (1981). Highlights and the perception of glossiness. Perception \& Psychophysics, 30, 407-410.

BiXBY, F. L. (1926). A phenomenological study of luster. Journal of General Psychology, 1, 136-174.

Evans, R. M. (1948). An introduction to color. New York: Wiley.

Evans, R. M. (1964). Variables of perceived color. Journal of the $O p$ tical Society of America, 54, 1465-1474.

Evans, R. M. (1974). The perception of color. New York: Wiley.

FLock, H. R. (1974). Stimulus structure in lightness and brightness experiments. In R. B. MacLeod \& H. L. Pick (Eds.), Perception: Essays in honor of James $J$. Gibson. Ithaca, NY: Comell University Press.

FLock, H. R. (1984). Illumination: Inferred or observed? Perception \& Psychophysics, 35, 293.

FLoCK, H. R., NoGuCHI, K. (1970). An experimental test of Jameson and Hurvich's theory of brightness contrast. Perception \& Psychophysics, 8, 129-136.

FLock, H. R., NusinowITZ, S. (1984). Visual structures for achromatic color perceptions. Perception \& Psychophysics, 36, 111-130.

FourT, L., HoworTH, R. M., \& RUTHERForD, M. B. (1954). Jmprovement of luster of cotton. Part III: Correlation of visual and physical aspects of luster. Textile Research Journal, 24, 61-66.

GELB, A. (1929). Die "Farbenkonstanz" der Sehdinge. In A. Bethe et al. (Eds.), Handbuch der normalen und pathologischen Physiologies (Vol. 12, Part 1). Berlin: Springer.

Gilchrist, A. L. (1980). When does perceived lightness depend on perceived spatial arrangement? Perception \& Psychophysics, 28. 527-538.

Heggelund, P. (1974). Achromatic color vison: 1. Perceptive variables of achromatic color. Vision Research, 14, 1071-1079.

HeLmHOLtz, H. vON (1925). Treatise on physiological optics (Vol. 3, J. C. P. Southall, Ed. and Trans.). Rochester, NY: Optical Society of America.

HELSON, H. (1938). Fundamental problems in color vision. I. The principle governing changes in hue, saturation, and lightness of nonselective samples in chromatic i"umination. Journal of Experimental Psychology, 23, 439-476. 
HeLson, H. (1943). Some factors and implications of color constancy. Journal of the Optical Society of America, 33, 555-567.

HeLson, H. (1964). Adaptation-level theory: An experimental and systematic approach to behavior. New York: Harper.

Henneman, R. H. (1935). A photometric study of the perception of object color. Archives of Psychology (New York), No. 179.

Hering, E. (1964). Outlines of a theory of the light sense (L. M. Hurvich \& D. Jameson, Trans.). Cambridge, MA: Harvard University Press. (Original work published 1905, 1907, 1911)

HsiA, Y. (1943). Whiteness constancy as a function of difference of illumination. Archives of Psychology (New York), No. 284.

HUNTER, R. S. (1958). Standardization of test for specular gloss of paper at $75^{\circ}$. Technical Association of the Pulp and Paper Industry, 41, 385-396.

HunTer, R. S. (1975). The measurement of appearance. New York: Wiley.

HUNTER, R. S., \& LoFLAND, C. A. (1956). A gloss test for waxed paper. Technical Association of the Pulp and Paper Industry, 39, 833-841.

INTERNATIONAL COMMission on IlLumination (1970). International lighting vocabulary (3rd ed.). Publication of the Commission Internationale De L'Eclairage, 17, 118.

Kardos, L. (1934). Ding und Schatten. Zeitschrift für PsychologieErganzungsband, No. 23.

KATZ, D. (1935). The world of colour (R. B. MacLeod and C. W. Fox, Trans.). London: Kegan, Trench, Trubner. (Original work, 2nd ed., published 1930)

KoFFKA, K. (1932). A new theory of brightness constancy: A contribution to a general theory of vision. Report of a joint discussion on vision held on Jan. 3, 1932 at the Imperial College of Sciences by the Physical and Optical Societies. Physical Society, London (Printed by the University Press, Cambridge), 182-188.
KoffKa, K. (1935). Principles of Gestalt psychology. New York: Harcourt Brace.

KozaKI, A. (1973). Perception of lightness and brightness of achromatic surface color and impression of illumination. Japanese Psychological Research, 15, 194-203.

LAND, E. H., \& MCCANN, J. J. (1971). Lightness and retinex theory. Journal of the Optical Society of America, 61, 1-11.

LIE, I. (1969). Psychophysical invariants of achromatic color vision: I. The multidimensionality of achromatic color experience. Scandinavian Journal of Psychology, 10, 167-175.

Matsuta, M., \& Kubota, T. (1981). Gloss measurement on painted surfaces. Japanese Joumal of Applied Physics, 20, 1145-1152.

NimerofF, I. (1952). Analysis of goniophotometric reflection curves. Journal of the Optical Society of America, 42, 579-583.

NoGUCHI, K., \& KozaKI, A. (1985). Perceptual scission of surfacelightness and illumination: An examination of the Gelb effect. Psychological Research, 47, 19-25.

O'DONNELL, F. X. D., \& BilLmeYer, F. W. (1986). Psychometric scaling of gloss. In J. J. Rennilson \& W. N. Hale (Eds.), Review and evaluation of appearance: Methods and techniques, STP-914. Philadelphia: American Society for Testing \& Materials.

WALLACH, H. (1948). Brightness constancy and the nature of achromatic colors. Journal of Experimental Psychology, 38, 310-324.

Wallach, H. (1963). The perception of neutral colors. Scientific American, 208, 107-116.

Werblin, F. S. (1973). The control of sensitivity in the retina. Scientific American, 228, 70-79.

(Manuscript received March 21, 1986; Revision accepted for publication April 16, 1987.) 